\title{
Corrigendum to 'A mechanistic study of the temperature dependence of the stress corrosion crack growth rate in SUS316 stainless steels exposed to pressurized water reactor primary water' [Acta Materialia 114 (2016) 15-24]
}

Martina Meisnar', Arantxa Vilalta-Clemente', Michael Moody', Koji Arioka², Sergio LozanoPerez"

1University of Oxford, Department of Materials, Parks Road, OX1 3PH, Oxford, UK

2Institute of Nuclear Safety Systems, Inc. (INSS), 64 Sata, Mihama-cho, Mikata-gun, Fuki, Mihama 919-1205, Japan

*corresponding author: telephone: +44 (0) 1865273795; e-mail: Sergio.lozano-perez@materials.ox.ac.uk

The authors regret that one of the main contributors to this paper was unintentionally omitted from the author list in the final version of the manuscript. The complete author list should read:

Martina Meisnar', Arantxa Vilalta-Clemente', Michael Moody', Angus J. Wilkinson', Koji Arioka $^{2}$, Sergio Lozano-Perez 